\title{
e-Migrinter
}

$18 \mid 2019$

L'ethnographie en migration(s)

\section{Ethnographie en migration, ethnographie des migrations}

Adelina Miranda

\section{CpenEdition}

Journals

Édition électronique

URL : https://journals.openedition.org/e-migrinter/1825

DOI : 10.4000/e-migrinter. 1825

ISSN : 1961-9685

Éditeur

UMR 7301 - Migrinter

Référence électronique

Adelina Miranda, «Ethnographie en migration, ethnographie des migrations », e-Migrinter [En ligne],

18 | 2019, mis en ligne le 12 septembre 2019, consulté le 20 mai 2021. URL : http://

journals.openedition.org/e-migrinter/1825; DOI : https://doi.org/10.4000/e-migrinter.1825

Ce document a été généré automatiquement le 20 mai 2021.

Tous droits réservés 


\title{
Ethnographie en migration, ethnographie des migrations
}

\author{
Adelina Miranda
}

1 Au cours des dernières années, le débat autour de l'ethnographie s'est distancé de ses premiers questionnements méthodologiques pour interroger les bases épistémologiques de la connaissance. Qu'elle soit considérée comme l'expression d'une participation engagée (Cefaï ; Amiraux, 2002), d'un engagement affectif sexuel (Broqua, 2000), d'un don/contredon (Bouillon, 2006) ou comme un espace de négociation des points de vue, l'ethnographie continue à être pensée comme étant à la base de la production d'un savoir élaboré à partir de la longue présence du chercheur sur le terrain, de son imprégnation et intégration progressive dans un espace relationnel d'interconnaissances et d'intersubjectivités. Toutefois, les questionnements reliant l'observation et la participation à l'objectivité, les positionnements du/de la chercheur.e aux différentes formes d'ethnocentrisme, la description à l'analyse, la comparaison à la généralisation, le particulier à l'universel, mettent en exergue les nombreuses tensions épistémologiques, méthodologiques et éthiques qui accompagnent l'adoption de cette approche.

2 Ces tensions ont été au cœur du débat qui a animé les chercheuses et chercheurs présents au colloque des trente ans du laboratoire Migrinter, et notamment lors de la journée dédiée aux travaux de jeunes chercheur.es en migrations intitulée "ethnographie et migrations". La journée avait pour ambition de rendre compte des apports épistémologiques et méthodologiques de l'ethnographie dans le champ des migrations internationales; et vice-versa, les innovations nécessaires à l'Ethnographe pour qu'elle ou il rende mieux compte des expériences de la migration.

3 Dans cette introduction, je propose d'opérer un retour critique sur ces problématiques au prisme des migrations en posant une question: qu'est-ce qu'on gagne à "ethnographier" les migrations? Pour y répondre, je reviendrai sur la fonction heuristique que recouvre le dépassement d'un "regard éloigné » notamment dans l'analyse de la question de l'altérité migrante; ensuite, j'aborderai la place que les multiples pratiques ethnographiques occupent dans l'étude des migrations et je 
questionnerai la « coprésence scientifique » du chercheur. Ce concept me permettra de situer ma réflexion dans un cadre critique des études migratoires et de plaidoyer pour une ethnographie des migrations. Celle-ci offre une démarche méthodologique et heuristique pour étudier la valeur conférée tant à l'altérité qu'à la mobilité, en tenant compte de la place que tous les sujets occupent dans les situations migratoires, tout en incluant dans cet espace les lieux de la recherche.

\section{Regards et altérités}

Un temps est désormais révolu : celui qui considérait l'ethnographie comme étant à la base d'une connaissance montant de l'observation concrète vers l'abstraction, définie comme étant la "vraie connaissance ». La " place auxiliaire » conférée à la pratique de terrain dans la construction du savoir anthropologique s'est basée sur le modèle de l'ethnographe solitaire, héroïque et malheureux (Weber, $2015:$ p. 249). Référée à une objectivité calquée sur les sciences naturelles, cette vision a laissé dans l'ombre les modalités au travers desquelles les chercheurs ont élaboré leur savoir opérant une transition des données plus concrètes vers des modèles abstraits, des échelles locales ou globales. De ce fait, l'abandon de la valeur scientifique accordée au «regard éloigné » a contribué à remettre en cause des hiérarchisations des savoirs (concret/ abstrait) basées sur une vision hiérarchisée du monde (mondes contemporain/ traditionnel, moderne/ sauvage etc.) et à accorder une grande importance tant à l'altérité qu'à la familiarité.

Ce passage, qui à partir des années 1960 s'est différemment déployé selon les contextes académiques nationaux (eux-mêmes liés aux traditions disciplinaires anthropologiques et coloniales), a cherché à comprendre si l'ethnographie était une pratique propre à l'anthropologie (L'Homme, 1986) et la place que l'altérité occupait dans cette pratique. Le retour sur l'histoire du travail de terrain depuis ses origines a montré que la professionnalisation du métier d'ethnographe n'a pas suivi un chemin linéaire et que les méthodes du folklore et de l'ethnologie se sont longtemps côtoyées (Cefai, 2003). Mais ce sont l'anthropologie des sociétés complexes (Lenclud, 1986), l'anthropologie du proche et l'anthropologie urbaine (Althabe, 1977; Petonnet, 1979) qui ont sorti la discipline du paradigme évolutionniste et traditionnel-centré qui l'avait structuré jusque-là. L'approche réflexive (Ghasarian, 2004), le caractère dialogique contenu dans la rencontre avec les sujets (Speber, 1982 ; Geertz, 1986), la mise en cause de «l'autorité ethnographique » (Marcus ; Clifford, 1986) ont définitivement libéré l'ethnographie d'un exotisme élaboré sur la représentation des autres à travers «un déni de cotemporalité » produisant un «discours allochronique » (Fabian, 1990) basé sur une opération de "désinterlocution" des individus (Chavier, 2011). Ce débat sur l'ethnographie s'insère dans un espace épistémique plus large qui réfléchit à l'élaboration des frontières entres les sciences sociales (Rapport de la commission Gulbenkian, 1996; Brailowsky; Inglebert, 2013), qui tient compte des circulations des savoirs et des pratiques au-delà des traditions nationales (Heilbron; Lenoir; Sapiro, 2004), des critiques féministes vis-à-vis d'une méthodologie androcentrée (Ramazanouglu; Holland, 2002) et de la place du colonialisme et des rapports de domination (Appadurai, 2005).

Ce débat a permis un retour critique sur la question de l'altérité. En effet, la définition classique de la relation ethnographique a souvent renforcé l'emphase anthropologique 
de la rencontre avec l'Autre. Selon la démarche "classique », l'ethnographe aurait dû se distancer de la "connaissance spontanée " à travers un passage graduel du particulier au général. Ce paradoxe épistémologique - être dedans et dehors, parvenir à une connaissance universelle sans l'Autre tout en s'appropriant ses catégories - se fonderait sur une procédure méthodologique permettant de dépasser la connaissance autochtone. La démarche ethnographique réflexive, en questionnant les bases de cette vision, a rappelé que l'Autre est défini et se définit à partir des valeurs et des paradigmes occidentaux. Elle englobe une hiérarchie entre celui qui pense l'Autre - le sujet de la pensée - et l'Autre qui est pensé - objet de la pensée - et elle occupe une place fondamentale dans la construction d'un savoir à vocation universelle mais qui, en effet, est fortement hiérarchisé (Saillant ; et al., 2011).

7 La remise en cause de l'articulation entre la distance géographique et l'altérité comme principe à la base de la construction d'un savoir objectif a eu des fortes implications pour le chercheur. La distance ethnologique est de ce fait de plus en plus considérée comme basée sur l'élaboration d'une «juste distance» (Bensa, 2013) ou bien d'une distanciation et d'un décentrement qui traversent un travail d'inter-subjectivation (Fassin ; Bensa, 2008). Comme le souligne Agier (2012) il s'agit d'assumer pleinement et rendre compte du caractère subjectif et relationnel de toute ethnographie, considérée non pas comme une identification mais comme une rencontre au sein de laquelle naît une compréhension.

8 Cette réflexivité ethnographique englobe donc une partie subjective sans pour autant se réduire à une opération solitaire psychologisante; elle analyse les modalités de production du savoir, l'enjeu de la neutralité scientifique et la déconstruction des modèles interprétatifs occidentaux-centrés. Cet « ethnocentrisme critique " repose sur une déconstruction des paradigmes et des catégories utilisées (De Martino, 1977), en situant l'échange entre chercheurs et sujets dans le temps et dans l'espace; cette approche localise et historicise la production des savoirs et elle alimente et renouvelle des nouveaux lieux du savoir (Bouillon ; et al., 2006) comme la migration.

En France, les premières recherches ethnographiques ont regardé les migrants comme des travailleurs (Barou, 1978; Catani, 1973), des « gens de banlieue» (Petonnet, 1978), des "étrangers" appartenant à des communautés (Terrain, 1986) ou des minorités urbaines (Raulin, 2009). Comme le soulignent Cuche et al. (2009), la spécificité méthodologique de l'anthropologie a contribué à une reconnaissance du point de vue de l'émigrant et de sa société d'origine. L'expérience ethnographique s'est donc présentée comme une condition pour une approche critique des migrations et la pratique réflexive comme une expression théorique permettant aussi de repositionner l'altérité migrante dans un débat plus vaste (Althabe, 1985 ; Sayad, 1997 - 1999). En effet, l'étude sur l'altérité migrante rappelle, d'un côté, que les altérités proches et éloignées sont reliées entre elles et que ce lien participe à la production d'un même ordre socio-politique et culturel et, d'un autre côté, que les catégories juridiques et/ou socio-économico-culturelles qui sont utilisées pour décrire l'altérité migrante englobent une valeur idéologique liée à une appartenance ethnico-nationale.

Cette mise en relation des formes d'altérités différentes à travers le prisme de l'immigration bouscule les anciennes hiérarchies des savoirs et contribue à la déconstruction d'une vision universaliste-occidentale de l'Autre permettant d'opérer trois constats majeurs. En premier lieu, elle invite à rendre accessoire la figure du migrant et à restituer les migrants dans une sorte de normalité. En deuxième lieu, elle 
permet d'observer que la mobilité n'est pas une condition qui s'ajoute ou qui est parallèle au monde sédentaire: la tension mobilité/sédentarité fait partie d'un processus plus large que Appadurai (2005) définit comme «la production de la localité ", c'est-à-dire de la manière dont les êtres humains (mobiles et sédentaires) exercent leurs capacités sociales, techniques et créatives. En troisième lieu, elle se pose comme la base d'une étude des migrations historicisée, localisée et relationnelle qui permet d'observer les processus au travers desquels les divers facteurs et motivations qui ordonnent les migrations se combinent et engendrent des morphologies migratoires évoluant dans le temps.

11 La production des discours sur l'altérité migrante renvoie à la mise en contact des sujets, migrant et non migrants. L'analyse de la manière dont les limites entre "nous " et les « autres » sont élaborées interroge l'importance des contacts socioculturels réels ou imaginés : ceux-ci produisent des frontières qui s'empilent, se décomposent et/ou ré-absorbent les précédentes en un jeu réversible et transmuable dans la recherche et la construction des multiples formes d'adhésion identitaire. Les nombreuses frontières qui apparaissent dans les espaces nationaux créent, en même temps, du lien social et de l'exclusion. Les questions migratoires sont en conséquence de plus en plus confrontées à l'actualité politique et nécessitent une réflexion éthique et épistémologique quant à l'accès au terrain (Clavé-Mercier ; Rigoni, 2017).

\section{Les multiples pratiques ethnographiques}

12 L'étude des migrations a contribué à la rupture d'une ethnographie traditionnelle sédentaire, qui considérait le cadre spatio-temporel de l'investigation comme étant stable, durable et fixe dans le temps et l'espace. Les approches transnationale (Glick Schiller ; et al., 1999), multisituée (Marcus, 1995) et multilocalisée (Miranda, 1997) ont contribué au développement d'une ethnographie des migrations critique et attentive aux mouvements des sujets, des objets et des représentations. Toutefois, cette réflexion ne considère que rarement la place du chercheur à partir de la multiplicité dans laquelle il est impliqué. Or, la relation ethnographique qui s'instaure entre sujets et chercheurs est autant traversée par la mobilité, par la pluri-appartenance sociale et culturelle que par la complexification du monde de la recherche et des conditions de travail auxquelles les chercheur.e.s sont confronté.e.s.

13 Pour avancer sur ce chemin réflexif, je propose de tenir compte de la « coprésence scientifique " vécue par les chercheur.e.s. Ce concept, qui s'inspire de celui de " coprésence migrante " (Berthomière; Hily, 2006), part du constat que les chercheur.e.s - comme tous sujets - sont imbriqués dans des situations plurielles, fluctuantes, réversibles; ils sont inscrits dans une diversité de mondes sociaux organisés par des processus structurels producteurs de hiérarchies et d'inégalités. L'usage de ce terme souligne, d'une part, la prise en compte des proximités et des frontières, des contiguïtés et des disjonctions, des bifurcations et des transitions qui se créent en pratiquant plusieurs terrains dans des temporalités identiques et/ou différentes. D'autre part, il intègre dans la recherche les dialectiques existant entre les mobilités et les sédentarités vécues par les chercheurs et la manière de gérer ses implications différentes. La "coprésence scientifique" permet enfin d'observer comment sur le terrain les appartenances multiples de/de la chercheur.e se combinent d'une manière changeante 
qui peut aller d'une grande implication à l'extériorité, en passant par toutes les positions intermédiaires.

Comment alors intégrer dans une démarche ethnographique la pluri-appartenance et la multi-localité vécues aussi par le chercheur? Comment tenir compte du croisement dans le temps et dans les lieux des savoirs des chercheur.e.s avec les savoirs des sujets de recherche? Ces deux questions en ouvrent une troisième: quelle est la place de l'appartenance de genre et de classe dans ces processus de connaissance?

15 Les auteur.e.s qui ont contribué à ce numéro abordent ces questions sous différents angles. Comme le montrent les textes présentés dans ce numéro, l'expérience du terrain se fonde sur une structure dialogique qui doit intégrer les regards multiples et diversifiés que les sujets portent sur le chercheur et aussi rendre compte de sa multipositionalité dans les champs académique mais également politique, social, économique et culturel. La production d'ethnographies multilocalisées s'est progressivement révélée être un outil qui permet de mieux saisir ces questionnements en tenant compte de la co-construction d'un savoir partagé. Magali N. Alloatti, dans sa recherche réalisée avec un groupe de femmes sur la pratique de la danse afrobrésilienne à Los Angeles, souligne le rôle joué par les " para-ethnographes ", ces sujets qui

" are individuals who are inserted in this system, whom must be characterized by their "own specificity, entangled in a particular set of forces [and by their] active sense making, the knowledge making complexified these subject's positions as knowledge makers ».

Néanmoins, la mise en place d'un mouvement de connaissance connectif implique l'engagement tant des chercheurs que des sujets et/ou des acteurs sociaux institutionnels et de la société civile participant au processus de recherche. La manière dont les engagements des uns et des autres se croisent et dialoguent reste l'enjeu principal pour la production d'une connaissance partagée afin d'élaborer un savoir non hégémonique, relationnel et partagé (Saillant; et al., 2011). Celui-ci implique la reconnaissance de la valeur heuristique de l'analyse de la pluralité des " points de vue » sur la réalité. Elle apparaît dans la capacité du chercheur à s'ouvrir à la confrontation afin de restituer le sens que les sujets attribuent à leurs pratiques et représentations depuis un point de vue situé, cet endroit réel et symbolique dans lequel ils agissent en tant qu'individus produits par les structures socio-économiques.

Ce processus de relativisation du savoir "scientifique" passe tout d'abord par une déconstruction des catégories d'analyse et il peut s'appuyer sur l'apport de nouvelles méthodologies. Pour Camille Gourdeau, l'enquête ethnographique menée au sein de cinq directions territoriales de l'office français de l'immigration et de l'intégration a permis de réfléchir sur la catégorie administrative de "primo-arrivant» et de mieux comprendre le profil et les carrières migratoires des étranger.e.s qui s'installent en France. De son côté, Amandine Desille s'appuie sur un film ethnographique réalisé lors des élections nationales en 2015 dans une ville périphérique du nord d'Israël pour démontrer comment le processus de distanciation peut se construire en combinant différents outils (filmer, observer, interviewer) tout en tenant compte du fait que le film ethnographique peut devenir autant un outil de collecte que de restitution. Les articles de Oriol Puig, de Armand Aupiais et de Aurore Vermylen apportent des réponses complémentaires aux ethnographies en situations migratoires sensibles (Bouillon ; et al., 2006). Pour le premier, l'honnêteté et la rigueur de l'ethnographie ont contribué à diminuer la relation de pouvoir inégal entre chercheur et collectif lors 
d'une recherche réalisée auprès des retournés nigériens de la Libye. Le second souligne la capacité de produire un "écart au regard de soi-même ", en passant par la pluralité des identifications dont le chercheur fait l'objet. Cette réflexion se base sur deux enquêtes sur les migrations des Pentecôtistes, l'une à Paris et l'autre à Istanbul. Cette condition a permis de mieux saisir les ressorts complexes et ambivalents qui accompagnent la recherche ethnographique. Pour la troisième, il s'agit de développer une anthropologie consciente de ses limites, en essayant de dépasser les «angles morts " par l'auto-analyse. À partir d'un terrain multi-situé réalisé dans la région des Grands Lacs en Afrique, au Kenya et en Amérique du Nord, l'auteure défend les apports de l'auto-analyse comme méthode de compréhension de nos terrains.

Bochra Manaï, à travers sa recherche réalisée à Montréal dans le quartier du PetitMaghreb, soulève l'épineuse question de l'incommode position occupée par les chercheur.e.s qui investissent des terrains d'enquête proche de leur appartenance, qu'elle soit réelle ou supposée. Le choix opéré par la chercheure de s'objectiver pour placer l'intersection des injustices urbaines et sociales dans un contexte d'islamophobie et l'option d'assumer une position engagée deviennent alors une opportunité de transformer le récit sur le territoire, qui permet d'interroger la démarche de recherche et la construction de la position « d'expert».

À travers leurs expériences, les auteur.e.s de ce numéro rendent compte des multiples pratiques ethnographiques et interrogent différemment la familiarité du chercheur avec le terrain, le rapport enquêté/enquêteur, et confirment que la recherche ethnographique est traversée par des formes de proximités et de distanciations relationnelles. Leurs points de vue situés soulignent que la recherche est une situation sociale qui ne se limite pas à l'expérience sur le terrain. Le dialogue scientifique construit et restitue le sens que les sujets attribuent à leurs actions, initiatives et représentations tout au long d'un processus; il contribue à la production de la connaissance des environnements dans lesquels les hommes et les femmes vivent et opèrent. Ces contributions confirment également que la place de l'ethnographe est toujours en devenir et que son implication et son engagement laissent ouvertes une série des questions, et notamment celle de l'écriture (Calzolaio ; et al., 2016). En même temps, elles éclairent l'hétérogénéité des trajectoires et des expériences et permettent de discuter des nouveaux outils conceptuels pour penser la mobilité (AterianusOwanga ; Musso, 2017) mais aussi le monde contemporain afin d'affirmer la recherche comme un droit humain pour favoriser l'exercice de la citoyenneté informée (Appadurai, 2013).

\section{BIBLIOGRAPHIE}

(1986) Anthropologie : état des lieux, L’Homme, vol. 26, n 97-98 [Disponible sur Internet].

(1986) Approches des communautés étrangères en France, Terrain, $n^{\circ} 7$ [Disponible sur Internet].

Althabe, Gérard (1977) Le procès au quotidien, Dialectiques, n² 21, pp. 67-77. 
Althabe, Gérard (1985) Production de l'étranger, xénophobie et couches populaires urbaines, L'Homme et la société, $\mathrm{n}^{\circ}$ 77-78, pp. 63-73.

Agier, Michel (2012) Penser le sujet, observer la frontière. Le décentrement de l'anthropologie, L'Homme, n' 203-204, pp. 51-75.

Agier, Michel (2015) Le dire-vrai de l'anthropologue. Réflexions sur l'enquête ethnographique du point de vue de la rencontre, des subjectivités et du savoir, ethnographiques.org, $\mathrm{n}^{\circ} 30$ (Mondes ethnographiques) [réf. du 25/02/2019] [Disponible sur Internet]

Appadurai, Arjun, (2005) Après le colonialisme. Les conséquences culturelles de la globalisation, Paris, Payot, $329 \mathrm{p}$.

Appadurai, Arjun, (2013) Condition de l'homme global, Paris, Payot $421 \mathrm{p}$.

Aterianus-Owanga, Alice; Musso, Sandrine (2017) Introduction. Anthropologie et migrations : mises en perspective, Lectures anthropologiques, $\mathrm{n}^{\circ} 3$ [Disponible sur Internet].

Barou, Jacques (1978) Les travailleurs africains en France, Présence Africaine, n 105-106, pp. 61-89.

Bensa, Alban (1995) De la relation ethnographique, Enquête, n 1, pp. 131-140 [Disponible sur internet] :

Berthomière, William ; Hily, Marie-Antoinette (2006) Décrire les migrations internationales. Les expériences de la co-présence, Revue européenne des migrations internationales, vol. $22, \mathrm{n}^{\circ} 2$, pp. 67-82 [Disponible sur Internet].

Bouillon, Florence (2006) Pourquoi accepte-t-on d'être enquêté ? Le contre-don, au cœur de la relation ethnographique, in Bouillon, F. ; Fresia, M. ; Tallio, V. (eds.) Terrains sensibles. Expériences actuelles de l'anthropologie, Paris, CEA/EHESS, pp. 75-95.

Bouillon, Florence ; Fresia, Marion ; Tallio, Virginie (eds) (2006) Terrains sensibles. Expériences actuelles de l'anthropologie, Paris, Éditions CEA/EHESS, 208 p.

Brailowsky, Yan ; Inglebert, Hervé (dir.) (2013) 1970-2010. Les sciences de l'Homme en débat, Nanterre, Presses universitaires de Paris Ouest, 489 p.

Broqua, Christophe (2000) Enjeux des méthodes ethnographiques dans l'étude des sexualités entre hommes, Journal des anthropologues, $\mathrm{n}^{\circ}$ 82-83, pp. 129-155 [Disponible sur Internet].

Calzolaio, Chiara ; Colombo, Pamela ; Makaremi, Chowra (2016) Ethnographies politiques de la violence. Introduction, Cultures \& Conflits, $\mathrm{n}^{\circ}$ 103-104, pp. 7-13 [Disponible sur Internet].

Catani, Maurizio (1973) L'alphabétisation des travailleurs immigrés : une relation dominant-dominé, Paris, Tema Éditions, 386 p.

Cefaï, Daniel (dir.) (2003) L'enquête de terrain, Paris, La Découverte, 624 p.

Cefaï, Daniel ; Valérie, Amiraux (2002) Les risques du métier. Engagements problématiques en sciences sociales. Partie 1, Cultures \& Conflits, $n^{\circ}$ 47, pp. 15-48 [Disponible sur Internet].

Chauvier, Eric (2011) Anthropologie de l'ordinaire. Une conversion du regard, Toulouse, Anacharsis, $170 \mathrm{p}$.

Clavé-Mercier, Alexandra ; Rigoni, Isabelle (2017) Enquêter sur les migrations : une approche qualitative, Migrations Société, n 167, pp. 15-28.

Clifford, James ; Marcus, Georges (eds.) (1986) Writing Culture, Berkeley, University of California Press, $305 \mathrm{p}$. 
Cuche, Denys ; Kuczynski, Liliane ; Raulin, Anne ; Razy, Élodie (2009) Éditorial, Revue européenne des migrations internationales, vol. 25, $\mathrm{n}^{\circ}$ 3, pp. 7-12 [Disponible dur Internet].

De Martino, Ernesto (2002) La fine del mondo, Turin, Einaudi, 724 p.

Fabian, Johannes (2014) Time and the Other. How Anthropology Makes Its Objects, Columbia University Press, $272 \mathrm{p}$.

Fassin, Didier ; Bensa, Alban (2004) Les politiques de l'enquête, Paris, La Découverte, 336 p.

Geertz, Clifford (1986) Savoir local, savoir global. Les lieux du savoir, Paris, Presse universitaire de France, $293 \mathrm{p}$.

Glick Schiller, Nina ; Basch, Linda ; Blanc-Szanton, Cristina (1992) Transnationalism : A New Analytic Framework for Understanding Migration, Annals of the New York Academy of Science, $\mathrm{n}^{\circ} 645$, pp. 1-24.

Ghasarian, Christian (2004) De l'ethnographie à l'anthropologie réflexive, Paris, Armand Colin, 249 p. Heilbron, Johan ; Lenoir, Remi ; Sapiro, Gisèle (eds.) (2004) Pour une histoire des sciences sociales, Paris, Fayard, 402 p.

Kilani, Mondher (2011) Antropologia. Dal locale al globale, Bari, Édition Dedalo, 480 p.

Lenclud, Gerard (1986) En être ou ne pas en être : L'anthropologie sociale et les sociétés complexes, L'Homme, vol. 26, n 97-98, pp. 143-153.

Marcus, Georges (1995) Ethnography in/of the World System : The Emergence of Multi-sites Ethnography, Annual Review of Anthropology, vol. 24, pp. 95-117.

Miranda, Adelina (1997) Migrants et non-migrants d'une commune italienne, Paris, L'Harmattan, $188 \mathrm{p}$.

Petonnet, Colette (1979) On est tous dans le brouillard : essai d'ethnologie urbaine, Paris, CTHS, 520 p.

Ramazanouglu, Caroline ; Holland, Janet (2002) Feminist Methodology: Challenges and Choices, Londre, Sage publications, $144 \mathrm{p}$.

Raulin, Anne (2009) Minorités urbaines : des mutations conceptuelles en anthropologie, Revue européenne des migrations internationales, $\mathrm{n}^{\circ} 25, \mathrm{pp}$. 33-51.

Rapport de la commission Gulbenkian (1996) Ouvrir les sciences sociales, Paris, Descartes \& Cie, $177 \mathrm{p}$.

Razy, Élodie ; Baby-Collin, Virginie (2011) La famille transnationale dans tous ses états, Autrepart, $\mathrm{n}^{\circ}$ 57-58, pp. 7-22.

Speber, Dan (1982) Le Savoir des anthropologues DES, Paris, Hermann, 140 p.

Saillant, Francine ; et al. (eds.) (2011) Manifeste de Lausanne. Pour une anthropologie non hégémonique, Montréal, Liber, 143 p.

Sayad, Abdelmalek (1997) L'immigration ou les paradoxes de l'altérité, Paris-Bruxelles, De Boek Université, $331 \mathrm{p}$.

Sayad, Abdelmalek (1999) La double absence. Des illusions de l'émigré aux souffrances de l'immigré, Paris, Seuil, 437 p.

Weber, Florence (2015) Brève Histoire de l'anthropologie, Paris, Flammarion, 354 p. 


\section{AUTEUR}

\section{ADELINA MIRANDA}

Professeure d'Anthropologie, Directrice de Migrinter - UMR 7301, CNRS / Université de Poitiers ;

Fellow Institut Convergence Migration

adelina.miranda@univ-poitiers.fr 